\title{
Role of ADAMTS13 in diet-induced liver steatosis
}

\author{
LOTTE GEYS $^{1}$, ELIEN ROOSE ${ }^{2}$, KAREN VANHOORELBEKE ${ }^{2}$, \\ PIERRE BEDOSSA $^{3}$, ILSE SCROYEN ${ }^{1}$ and H. ROGER LIJNEN ${ }^{1}$ \\ ${ }^{1}$ Department of Cardiovascular Sciences, Center for Molecular and Vascular Biology, University of Leuven, \\ B-3000 Leuven; ${ }^{2}$ Laboratory for Thrombosis Research, Department of Chemistry, \\ University of Leuven Kulak Campus Kortrijk, B-8500 Kortrijk, Belgium; ${ }^{3}$ Department of \\ Pathology, Physiology and Imaging, Université Paris Diderot, 75013 Paris, France
}

Received January 11, 2017; Accepted April 28, 2017

DOI: $10.3892 / \mathrm{mmr} .2017 .6714$

\begin{abstract}
Previous studies, predominantly based on increased or decreased plasma levels, have reported conflicting data on a potential functional role of ADAMTS13 in the pathogenesis of liver diseases, including non-alcoholic steatohepatitis (NASH). The aim of the current study was to evaluate whether ADAMTS13 deficiency affects development of NASH. Therefore, male wild-type (WT) and Adamts13 deficient (Adamts13 ${ }^{-/}$) mice were kept on a steatosis-inducing diet devoid of methionine and choline (MCD) or a control diet (MCC) for 4 weeks. Induction of NASH did not affect plasma ADAMTS13 antigen levels of WT mice. MCD as compared with MCC feeding resulted in reduced body and liver weight with no differences between the genotypes. Plasma levels of the liver enzymes AST and ALT were significantly higher for MCD vs. MCC fed Adamts13 $3^{--}$and WT mice, however were not different between the genotypes. Liver triglyceride levels were also higher after MCD feeding, but were not different between WT and Adamts $13^{-/-}$mice. Adamts $13^{-/-}$mice on the two diets exhibited higher insulin sensitivity when compared with WT mice. On the MCC diet, the genotype did not show clear histological abnormalities in the liver, whereas severe steatosis and fibrosis were observed on MCD diet, however were comparable for both genotypes. This was supported by comparably enhanced hepatic expression in the two genotypes on MCD diet of the steatosis marker CD36 and of the fibrosis marker tissue inhibitor of metalloproteinase 1 . Thus, the results of the current study do not support a functional role of ADAMTS13 in this murine model of NASH.
\end{abstract}

Correspondence to: Professor H. Roger Lijnen, Department of Cardiovascular Sciences, Center for Molecular and Vascular Biology University of Leuven, Campus Gasthuisberg, CDG, Herestraat 49, Box 911, B-3000 Leuven, Belgium

E-mail: roger.lijnen@kuleuven.be

Key words: a disintegrin and metalloproteinase with thrombospondin type-1 motif, member 13, liver steatosis, mouse model, diet, non-alcoholic fatty liver disease

\section{Introduction}

Non-alcoholic fatty liver disease (NAFLD) is characterized by excessive fat accumulation in the liver of patients without a history of alcohol abuse. NAFLD is classified into simple steatosis and non-alcoholic steatohepatitis (NASH). In patients with NASH, in addition to steatosis, additional intralobular inflammation and hepatocellular ballooning are observed, frequently with progressive fibrosis (1). Over time, NASH may progress to liver cirrhosis and hepatocellular carcinoma (2-4). It is recognized that the increased prevalence of obesity and metabolic syndrome is paralleled by an increase in NAFLD $(5,6)$, as up to $80 \%$ of obese subjects suffer from NAFLD (7). Worldwide, approximately $20 \%$ of all adults have NAFLD and 2-3\% suffer from NASH (8).

ADAMTS13 (a disintegrin and metalloproteinase with thrombospondin type-1 motif, member 13) is a proteinase that specifically cleaves multimeric von Willebrand factor (VWF), thereby preventing accumulation of ultralarge VWF multimers and subsequent platelet clumping and formation of microthrombi that disturb the microcirculation and impair oxygen supply to organs (9). ADAMTS13 is predominantly produced by stellate cells in the liver (10), of which proliferation contributes to steatohepatitis and fibrosis. This is compatible with enhanced ADAMTS13 antigen and activity levels observed in rats suffering from diet-induced steatosis and fibrosis (11). Several previous studies have, however, reported conflicting data on a potential functional role of ADAMTS13 in the pathogenesis of liver diseases [reviewed in (12)]. A beneficial effect of ADAMTS13 activity was observed on liver disease severity, and increased ADAMTS13 activity was associated with an improved prognosis of liver cirrhosis (13), alcoholic hepatitis (14) and hepatic veno-occlusive disease (15). In contrast, a detrimental effect of ADAMTS13 was reported on hepatocellular carcinoma risk in patients suffering from chronic liver disease (16). Thus, it remains unclear whether NASH is associated with increased or decreased levels of ADAMTS13, nor whether ADAMTS13 serves a functional role in its development. In order to resolve these apparent contradictions, we have subjected wild-type and Adamts 13 deficient mice to a steatosis-inducing diet. 


\section{Materials and methods}

Animal model. Male $(\mathrm{n}=2)$ and female $(\mathrm{n}=2)$ heterozygous Adamts $13^{+/}$mice were provided by Professor David Ginsburg (Howard Hughes Medical Institute, University of Michigan, Ann Arbor, MI, USA) and were crossed to generate Adamts 13 deficient (Adamts $13^{-1}$ ) and wild-type (WT) littermates (genetic background, C57B16/Jx129X1/SvxCASA/RK). Male Adamts13 $3^{-/}(\mathrm{n}=20)$ and WT $(\mathrm{n}=10)$ mice were used and genotyped as previously described $(17,18)$. The mice were kept from the age of 5 weeks in individual micro-isolation cages on a $12 \mathrm{~h}$ day/night cycle and fed for 4 weeks with a lipogenic diet devoid of methionine and choline (MCD; \#02960034; MP Biomedicals, Illkirch Cedex, France) or the MCD diet supplemented with $3 \mathrm{~g} / \mathrm{kg}$ DL-methionine and $2 \mathrm{~g} / \mathrm{kg}$ choline chloride (control diet; MCC; \#02960414; MP Biomedicals) ( $\mathrm{n}=10$ or 5 for Adamts $13^{-/-}$or WT mice on each diet, respectively). The starting weight of the mice was $26.4 \pm 0.8 \mathrm{~g}$ or $27.0 \pm 1.0 \mathrm{~g}$ for WT mice on MCD or MCC diets, respectively, and $26.0 \pm 0.5 \mathrm{~g}$ or $25.4 \pm 0.7 \mathrm{~g}$ for Adamts $13^{-/}$mice on MCD or MCC diets, respectively. Water was available ad libitum, room temperature and relative humidity were $23^{\circ} \mathrm{C}$ and $26 \%$, respectively. Body weight and food intake were measured at weekly intervals. At the end of the diet, following fasting for $6 \mathrm{~h}$, blood was taken from the tail of unanesthetized mice for determination of blood glucose concentrations using the Accu-chek Performa meter and blood glucose test strips (Roche Diagnostics, Basel, Switzerland). At the end of the experiment, the mice were sedated with $60 \mathrm{mg} / \mathrm{kg}$ pentobarbital (Nembutal; Ceva Santé Animale, Libourne, France) and blood was taken from the retro-orbital sinus on trisodium citrate $(0.01 \mathrm{M})$, prior to sacrifice by cervical dislocation. Platelets were counted (Cell Dyn 3200R; Abbott Diagnostics, Illinois, USA). Livers, subcutaneous (SC) and gonadal (GN) adipose tissues were removed and weighed. Portions were used for RNA or protein extraction or were fixed in $4 \%$ formaldehyde for histological analysis.

All animal experiments were approved by the University of Leuven Ethical Committee (P158-2011) and performed in accordance with the National Institutes of Health Guide for the Care and Use of Laboratory Animals (19) and the EU Directive 2010/63/EU for animal experiments.

Metabolic and inflammatory parameters. Total, high density lipoprotein (HDL) and low density lipoprotein (LDL) cholesterol, triglycerides, alkaline phosphatases, alanine aminotransferase (ALT) and aspartate aminotransaminase (AST) levels in plasma were evaluated using routine clinical assays. Insulin levels in plasma were determined by ELISA (Ultrasensitive Mouse Insulin ELISA 10-1249-01; Mercodia AB, Uppsala, Sweden). The homeostasis model assessment of insulin resistance (HOMA-IR) was determined using the formula: [Fasting plasma insulin $(\mathrm{ng} / \mathrm{ml}) \mathrm{x}$ fasting blood glucose $(\mathrm{mg} / \mathrm{dl})] / 405$. Liver tissue extracts were prepared by adding alcoholic $\mathrm{KOH}(30 \%)$ to a sample of $30 \mathrm{mg}$. Samples were incubated overnight at $55^{\circ} \mathrm{C}$ to digest the tissue, and $1 \mathrm{M}$ $\mathrm{MgCl}_{2}$ was added to the digested sample (1:1 vol/vol), mixed well, incubated on ice for $10 \mathrm{~min}$ and centrifuged for $30 \mathrm{~min}$ at $13,523 \mathrm{x} \mathrm{g}$ and room temperature. The supernatant was analyzed using the Triglycerides FS* kit (DiaSys Diagnostic Systems GmbH, Holzheim, Germany).
Table I. Markers detected by reverse transcription-quantitative polymerase chain reaction using TaqMan gene expression assays.

\begin{tabular}{ll}
\hline Gene & \multicolumn{1}{c}{ Assay } \\
\hline F4/80 & Mm00802529_m1 \\
TNF- $\alpha$ & Mm00443258_m1 \\
MCP-1 & Mm00441242_m1 \\
IL-6 & Mm0446190_m1 \\
Arginase & Mm00475988_m1 \\
Mannose receptor & Mm00485148_m1 \\
FAS & Mm01253300_g1 \\
CD36 & Mm00432403_m1 \\
PPAR- $\alpha$ & Mm00440939_m1 \\
TIMP-1 & Mm0441818_m1 \\
PAI-1 & Mm0435860_m1 \\
$\alpha-S M A$ & Mm00725412_s1 \\
Col1a1 & Mm00801666_g1 \\
TGF- $\beta$ & Mm01178820_m1 \\
$\beta$-actin & Mm01205647_g1 \\
\hline
\end{tabular}

All assays were purchased from Life Technologies (Thermo Fisher Scientific, Inc., Waltham, MA, USA). TNF- $\alpha$, tumor necrosis factor $\alpha$; MCP-1, monocyte chemoattractant protein 1; IL-6, interleukin 6; FAS, Fas cell surface death receptor; CD36, cluster of differentiation; PPAR- $\alpha$, peroxisome proliferator-activated receptor $\alpha$; TIMP-1, tissue activator of metalloproteinase 1; PAI-1, plasminogen activator inhibitor 1 ; $\alpha$-SMA, $\alpha$-smooth muscle actin; Colla1, collagen type I $\alpha 1$ chain; TGF- $\beta$, transforming growth factor $\beta$.

Histological and microscopic analysis. Liver samples were fixed in $4 \%$ buffered formaldehyde and embedded in paraffin. Sections $(4 \mu \mathrm{m})$ were stained with hematoxylin-eosin (H\&E) or Picrosirius Red to assess steatosis or fibrosis, respectively. All liver biopsies were analyzed by an expert liver pathologist, blinded to the genotype and diet. Steatosis and fibrosis were diagnosed and semiquantitatively scored according to the NASH-Clinical Research Network criteria $(20,21)$. Hepatocyte ballooning was classified as 0 (none), 1 (few) or 2 (numerous cells/prominent ballooning). Foci of lobular inflammation were scored as 0 (no foci), 1 ( $<2$ foci per $\times 200$ field), 2 ( $2-4$ foci per x200 field) and 3 ( $>4$ foci per x200 field). Fibrosis was scored as stage F0 (no fibrosis), stage F1a (mild, zone 3, perisinusoidal fibrosis), stage F1b (moderate, zone 3, perisinusoidal fibrosis), stage F1c (portal/periportal fibrosis), stage F2 (perisinusoidal and portal/periportal fibrosis), stage F3 (bridging fibrosis) and stage F4 (cirrhosis). Severity of the disease was assessed using the NAFLD activity score (NAS) as the unweighted sum of scores of steatosis, hepatocyte ballooning and lobular inflammation (22). Percentage of fibrosis was quantitated by morphometry from digitalized Picrosirius Red stained sections (23).

RNA extraction and expression analysis. RNA extraction from livers was performed using the RNeasy Mini kit (Qiagen, Basel, Switzerland) according to the manufacturer's protocol. A total of $10 \mathrm{ng} / \mu \mathrm{l}$ RNA was reverse transcribed into cDNA using the Multiscribe $^{\mathrm{TM}}$ Reverse Transcriptase kit (Life Technologies; 
A

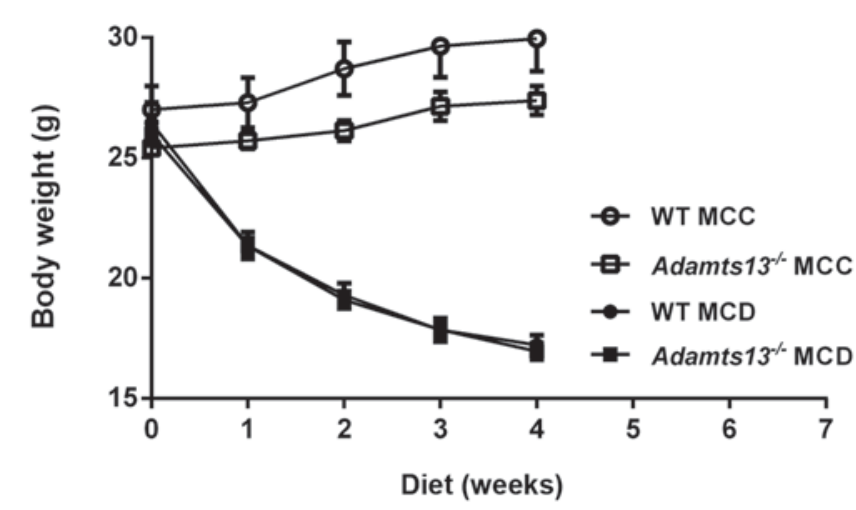

B

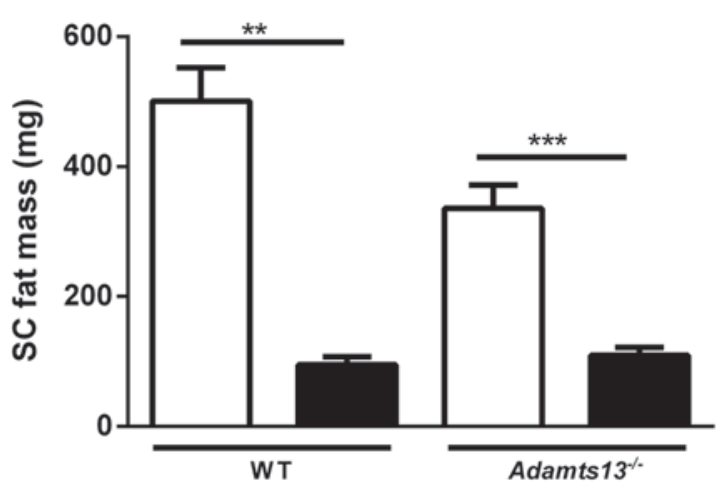

C

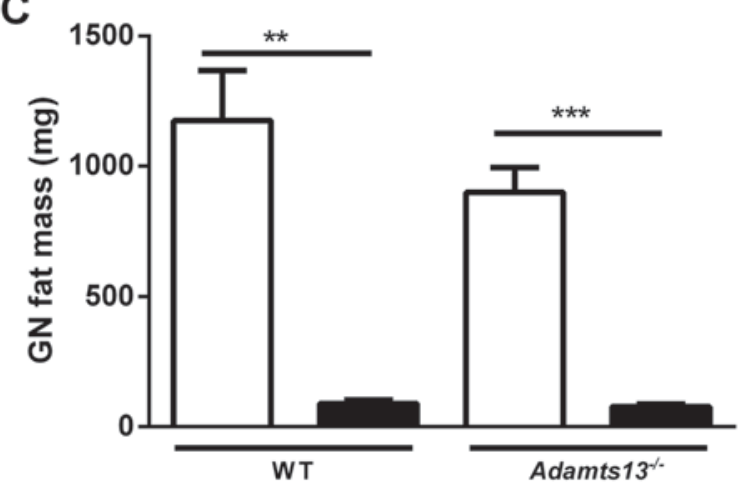

E

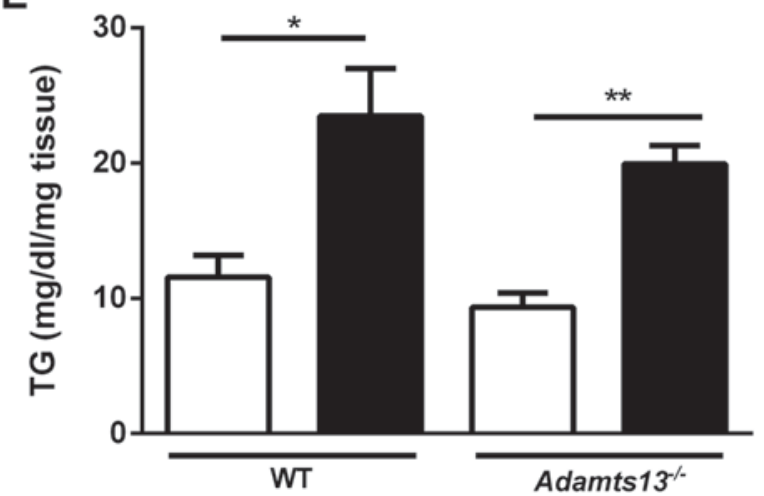

D

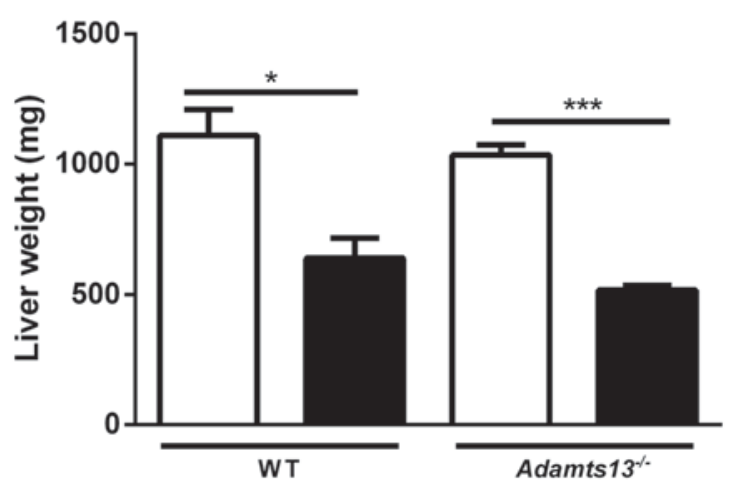

F

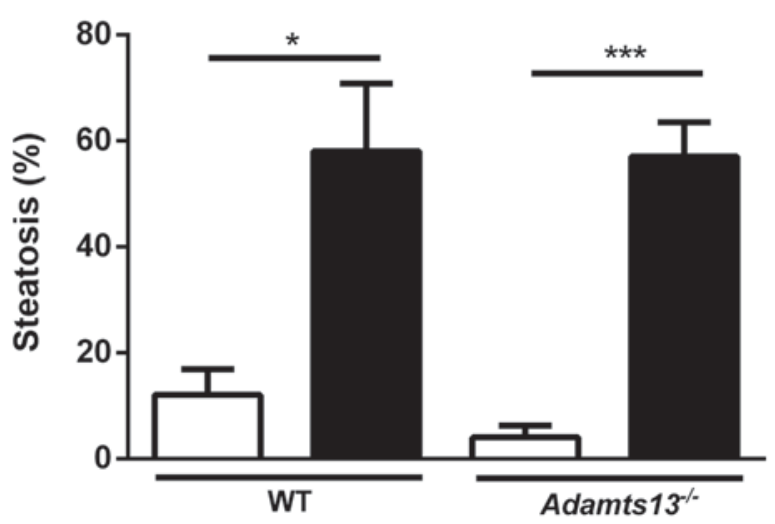

Figure 1. Effect of MCD and MCC diets on body weight and liver of WT and Adamts $13^{-/}$mice. (A) Body weight evolution and weight of (B) SC fat, (C) GN fat and (D) liver of mice kept on MCD (black symbols and bars) or MCC (white symbols and bars) diets for 4 weeks. (E) Liver TG levels and (F) quantitation of liver steatosis. Data are presented as the mean \pm standard error of 10 (Adamts $13^{-/-}$) or $5(\mathrm{WT})$ experiments for each diet. ${ }^{*} \mathrm{P}<0.05,^{* *} \mathrm{P}<0.01$ and ${ }^{* * * *} \mathrm{P}<0.001$ MCD vs. MCC. MCD, methionine and choline deficient diet; MCC, control diet; WT, wild-type; Adamts $13^{-/-}$, a disintegrin and metalloproteinase with thrombospondin type-1 motif, member 13 deficient; SC, subcutaneous; GN, gonadal; TG, triglyceride.

Thermo Fisher Scientific, Inc., Waltham, MA, USA), according to the manufacturer's protocol. Reverse transcription-quantitative polymerase chain reaction was completed in the ABI 7500 Fast Sequence detector (Life Technologies; Thermo Fisher Scientific, Inc.) to detect the markers listed in Table I. Thermocycling conditions were as follows: Fast mode run of 40 cycles of $20 \mathrm{sec}$ at $95^{\circ} \mathrm{C}, 3 \mathrm{sec}$ at $95^{\circ} \mathrm{C}$ and $30 \mathrm{sec}$ at $60^{\circ} \mathrm{C}$. The reaction mixture contained $5 \mu \mathrm{l}$ TaqMan Fast Universal PCR Master Mix (2X; Life Technologies; Thermo Fisher Scientific, Inc.), $0.5 \mu 1$ TaqMan Gene Expression assay (see Table I; Life Technologies;
Thermo Fisher Scientific, Inc.) and $2 \mu \mathrm{l}$ cDNA diluted with RNase-free water to a total volume of $10 \mu \mathrm{l}$. For ADAMTS13 expression, probe $(0.2 \mu \mathrm{l} ; 1$ :10 diluted probe; Life Technologies; Thermo Fisher Scientific, Inc.), and forward and reverse primers (0.3 $\mu \mathrm{l} ;$ 1:10 diluted primer; Life Technologies; Thermo Fisher Scientific, Inc.) were used instead of TaqMan Gene Expression assays. The sequences of the probe, and forward and reverse primers for ADAMTS13 expression were as follows: Forward primer, GGAGCCCAAGGATGTGTGTCTT; reverse primer, TCTCTGGAGGTGAGAGGGAGGAT; and probe, 
6FAM (reporter dye) CTTGGCCACCATGCT MGBNFQ (non-fluorescent quencher with maximum sensitivity). Analyses were performed using the $\Delta \Delta \mathrm{Cq}$ method (24) using the 7500 System SDS software (Life Technologies; Thermo Fisher Scientific, Inc.). Normalization was conducted to correct for fluctuations caused by sample differences. Fold changes for MCD diet fed mice were calculated as $2^{-\Delta \Delta C q}$ relative to the MCC diet. $\beta$-actin was used as the housekeeping gene.

VWF and ADAMTS13 antigen levels. Murine VWF and ADAMTS13 antigen levels in the plasma were measured using ELISA assays made in the Laboratory for Thrombosis Research (Department of Chemistry, University of Leuven Kulak Campus, Kortrijk, Belgium), as previously described $(25,26)$.

Statistical analysis. Data are presented as the means \pm standard error of. Statistical significance between groups was analyzed with the non-parametric Mann-Whitney U test. Analysis of the data was performed using Prism, version 6 (GraphPad Software, Inc., San Diego, CA, USA). P $<0.05$ was considered to indicate a statistically significant difference.

\section{Results}

The effects of the MCD diet were determined on body weight and liver function in WT and Adamts $13^{-/}$mice (Fig. 1) . Feeding WT and Adamts $13^{-1-}$ mice with the MCD diet for 4 weeks, as compared with the control MCC diet, resulted in rapid and progressive weight loss for the two genotypes (Fig. 1A). Food intake was comparable for WT and Adamts $13^{-/-}$mice kept on MCC (3.3 vs. $3.0 \mathrm{~g} / \mathrm{mouse} / \mathrm{day})$ or on MCD diet (2.4 vs. $2.2 \mathrm{~g} /$ mouse/day). At the end of the diets, total body weight was comparable for WT and Adamts $13^{-/}$mice on the MCC ( $30.0 \pm 1.4$ vs. $27.4 \pm 0.6 \mathrm{~g})$ and MCD diets $(17.2 \pm 0.4$ vs. $17 \pm 0.4 \mathrm{~g}$ ). As predicted, the SC and GN fat mass levels were significantly reduced for in each genotype comparing the MCD and MCC diets (Fig. 1B and C).

For WT mice, plasma ADAMTS13 antigen levels were not significantly different between the MCC and MCD diets ( $183 \pm 12$ vs. $205 \pm 17 \%$ of pooled plasma), and relative expression of Adamts $13 \mathrm{mRNA}$ in the liver was also comparable on the two diets $(0.91 \pm 0.07$ vs. $1.0 \pm 0.05)$. VWF antigen levels were comparable for the genotypes on either diet (Table II). However, platelet counts were significantly lower for Adamts $13^{-/}$fed the MCD when compared with MCC, however this was not observed in WT mice (Table II). Analysis of plasma metabolic parameters (Table II) identified for MCD vs. MCC diets for the two genotypes: i) Lower glucose levels; ii) lower total and HDL cholesterol levels; iii) higher LDL cholesterol levels ( $\mathrm{P}<0.05$ for Adamts $13^{-/}$mice only); and iv) comparable triglyceride levels. Insulin levels were markedly lower in MCD vs. MCC diet (significant for the Adamts $13^{-/-}$mice due to small sample sizes for WT mice), and were reduced for Adamts $13^{-/}$vs. WT mice on MCC. Thus, HOMA-IR identified higher insulin sensitivity for Adamts $13^{--}$mice on the MCC diet (Table II).

Total liver weight was reduced upon MCD feeding, however was not affected by the genotype (Fig. 1D). The liver enzymes AST and ALT were markedly higher in MCD fed as compared with MCC fed mice, however were not different between genotypes (Table II). Liver triglyceride levels were additionally
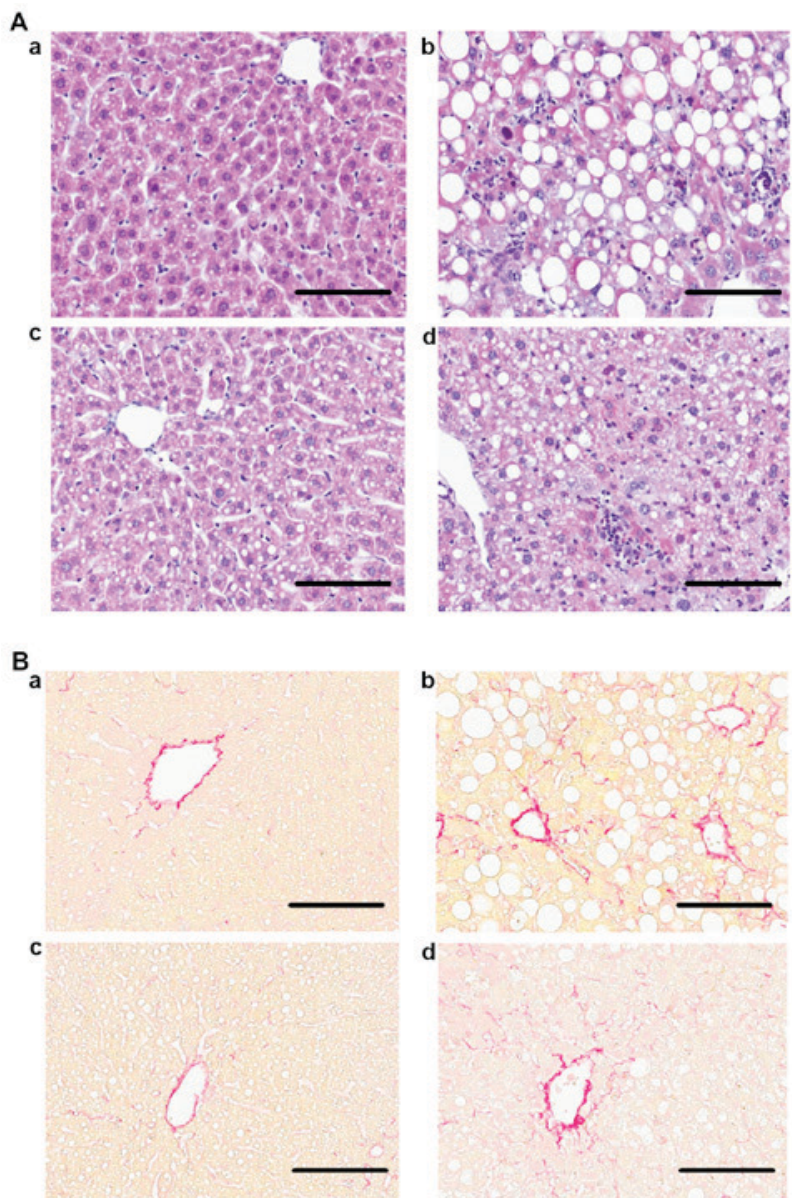

d

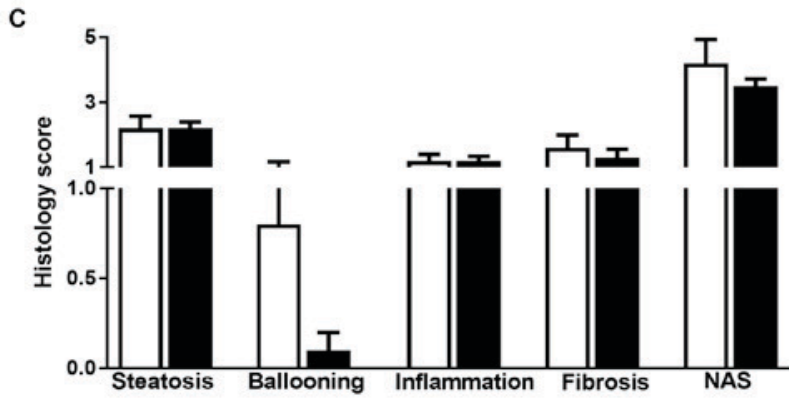

Figure 2. MCD diet-induced steatohepatitis in WT and Adamts $13^{-/-}$mice. (A) Hematoxylin and eosin and (B) Picrosirius Red staining of liver sections of (a and b) WT and (c and d) Adamts $13^{-/-}$mice kept on (a and c) MCC or (b and d) MCD diet for 4 weeks. Scale bars represent $100 \mu \mathrm{m}$. (C) Histological scoring of steatosis, hepatocyte ballooning, inflammation, fibrosis and NAS for mice maintained on a MCD diet. Data are presented as the mean \pm standard error of 10 (Adamts $13^{-/}$, black bars) or 5 (WT, white bars) determinations. MCD, methionine and choline deficient diet; WT, wild-type; Adamts $13^{-/-}$, a disintegrin and metalloproteinase with thrombospondin type-1 motif, member 13 deficient; MCC, control diet; NAS, nonalcoholic fatty liver disease activity score.

enhanced upon MCD feeding, however were not affected by the genotype (Fig. 1E).

The effects of the MCD diet were determined on steatohepatitis in WT and Adamts13-- mice (Fig. 2). H\&E staining of liver sections identified more pronounced steatosis in MCD as compared with MCC fed mice of both genotypes (Fig. 2A), as confirmed by quantitative analysis (Fig. 1F). Further histological analysis of liver sections identified that following 4 weeks of MCC diet, WT (3/5 with score 1) and Adamts $13^{-/}$(3/10 with 
Table II. Plasma levels of metabolic parameters, liver enzymes, endogenous VWF and platelet count of WT and Adamts13 ${ }^{-/-}$mice kept on MCC or MCD diets for 4 weeks.

\begin{tabular}{|c|c|c|c|c|}
\hline \multirow[b]{2}{*}{ Parameter } & \multicolumn{2}{|c|}{ WT } & \multicolumn{2}{|c|}{ Adamts $13^{-/-}$} \\
\hline & $\mathrm{MCC}$ & MCD & $\mathrm{MCC}$ & MCD \\
\hline Glucose (mg/dl) & $194 \pm 20.4$ & $94 \pm 8.98^{\mathrm{a}}$ & $177 \pm 6.09$ & $93 \pm 3.86^{b}$ \\
\hline Insulin (ng/ml) & $0.58 \pm 0.12$ & $0.17 \pm 0.07$ & $0.26 \pm 0.06^{c}$ & $0.05 \pm 0.01^{\mathrm{d}}$ \\
\hline HOMA-IR & $0.30 \pm 0.06$ & $0.05 \pm 0.02^{\mathrm{d}}$ & $0.12 \pm 0.03^{c}$ & $0.01 \pm 0.002^{\mathrm{a}}$ \\
\hline Triglycerides (mg/dl) & $45 \pm 4.6$ & $36 \pm 0.95$ & $35 \pm 1.7$ & $31 \pm 1.0^{c}$ \\
\hline Cholesterol (mg/dl) & $89 \pm 14$ & $31 \pm 5^{\mathrm{d}}$ & $84 \pm 5$ & $30 \pm 1^{\mathrm{e}}$ \\
\hline HDL cholesterol (mg/dl) & $82 \pm 13$ & $22 \pm 2^{\mathrm{d}}$ & $75 \pm 4^{c}$ & $17 \pm 1^{\mathrm{e}}$ \\
\hline LDL cholesterol (mg/dl) & $1.5 \pm 1.5$ & $7.5 \pm 0.9$ & $3.8 \pm 0.9$ & $7.0 \pm 0.9^{\mathrm{d}}$ \\
\hline Alkaline phosphatases (U/l) & $63 \pm 7.6$ & $76 \pm 12$ & $56 \pm 3.2$ & $77 \pm 3.0^{\mathrm{d}}$ \\
\hline $\operatorname{AST}(\mathrm{U} / \mathrm{l})$ & $58 \pm 13$ & $185 \pm 62.0^{\mathrm{d}}$ & $78 \pm 12$ & $173 \pm 14.4^{\mathrm{e}}$ \\
\hline ALT (U/l) & $22 \pm 2.0$ & $193 \pm 53.4^{\mathrm{a}}$ & $39 \pm 9.0$ & $227 \pm 24.0^{\mathrm{b}}$ \\
\hline Platelet count $\left(\mathrm{x} 10^{3} / \mu \mathrm{l}\right)$ & $917 \pm 84.3$ & $752 \pm 117$ & $829 \pm 25$ & $565 \pm 65^{\mathrm{a}}$ \\
\hline mVWF (\% to NMP) & $302 \pm 2.53$ & $250 \pm 38.9$ & $247 \pm 40.3$ & $168 \pm 37.5$ \\
\hline
\end{tabular}

Data are presented as the means \pm standard error of 10 Adamts $13^{-/}$or 5 WT experiments for each diet. ${ }^{\mathrm{a} P}<0.01$ and ${ }^{\mathrm{b}} \mathrm{P}<0.0001 \mathrm{MCD}$ vs. MCC fed mice; ${ }^{\mathrm{P}}<0.05$ Adamts $13^{-/-}$vs. WT mice; ${ }^{\mathrm{d}} \mathrm{P}<0.05$ and ${ }^{\mathrm{e}} \mathrm{P}<0.001 \mathrm{MCD}$ vs. MCC fed mice. WT, wild-type; Adamts $13^{-/-}$, a disintegrin and metalloproteinase with thrombospondin type-1 motif, member 13 deficient; MCD, methionine and choline deficient diet; MCC, control diet; HOMA-IR, homeostasis model assessment of insulin resistance; HDL, high density lipoprotein; LDL, low density lipoprotein; AST, aspartate aminotransaminase; ALT, alanine aminotransferase; mVWF, murine von Willebrand factor; NMP, normal mouse plasma.

Table III. Histological scoring of liver sections of mice maintained on MCC or MCD diet for 4 weeks.

\begin{tabular}{|c|c|c|c|c|}
\hline \multirow[b]{2}{*}{ Factor } & \multicolumn{2}{|c|}{$\mathrm{MCC}$} & \multicolumn{2}{|c|}{ MCD } \\
\hline & WT & Adamts $13^{--}$ & WT & Adamts $13^{-/-}$ \\
\hline \multicolumn{5}{|c|}{ Microvesicular steatosis } \\
\hline Score $0(<5 \%)$ & $2 / 5$ & $7 / 10$ & $0 / 5$ & $0 / 10$ \\
\hline Score $1(5-33 \%)$ & $3 / 5$ & $3 / 10$ & $1 / 5$ & $1 / 10$ \\
\hline Score $2(33-66 \%)$ & $0 / 5$ & $0 / 10$ & $2 / 5$ & $6 / 10$ \\
\hline Score $3(>66 \%)$ & $0 / 5$ & $0 / 10$ & $2 / 5$ & $3 / 10$ \\
\hline \multicolumn{5}{|c|}{ Hepatocyte ballooning } \\
\hline Score 0 & $5 / 5$ & $10 / 10$ & $2 / 5$ & $9 / 10$ \\
\hline Score 1 & $0 / 5$ & $0 / 10$ & $2 / 5$ & $1 / 10$ \\
\hline Score 2 & $0 / 5$ & $0 / 10$ & $1 / 5$ & $0 / 10$ \\
\hline \multicolumn{5}{|l|}{ Lobular inflammation } \\
\hline Score 0 & $5 / 5$ & $10 / 10$ & $0 / 5$ & $0 / 10$ \\
\hline Score 1 & $0 / 5$ & $0 / 10$ & $4 / 5$ & $8 / 10$ \\
\hline Score 2 & $0 / 5$ & $0 / 10$ & $1 / 5$ & $2 / 10$ \\
\hline Score 3 & $0 / 5$ & $0 / 10$ & $0 / 5$ & $0 / 10$ \\
\hline \multicolumn{5}{|l|}{ Fibrosis } \\
\hline F0 & $5 / 5$ & $10 / 10$ & $1 / 5$ & $1 / 10$ \\
\hline Stage F1a & $0 / 5$ & $0 / 10$ & $0 / 5$ & $6 / 10$ \\
\hline Stage F1b & $0 / 5$ & $0 / 10$ & $4 / 5$ & $2 / 10$ \\
\hline Stage F1c & $0 / 5$ & $0 / 10$ & $0 / 5$ & $0 / 10$ \\
\hline Stage F2 & $0 / 5$ & $0 / 10$ & $0 / 5$ & $1 / 10$ \\
\hline
\end{tabular}

MCD, methionine and choline deficient diet; MCC, control diet; WT, wild-type; Adamts $13^{-/}$, a disintegrin and metalloproteinase with thrombospondin type-1 motif, member 13 deficient. 
A

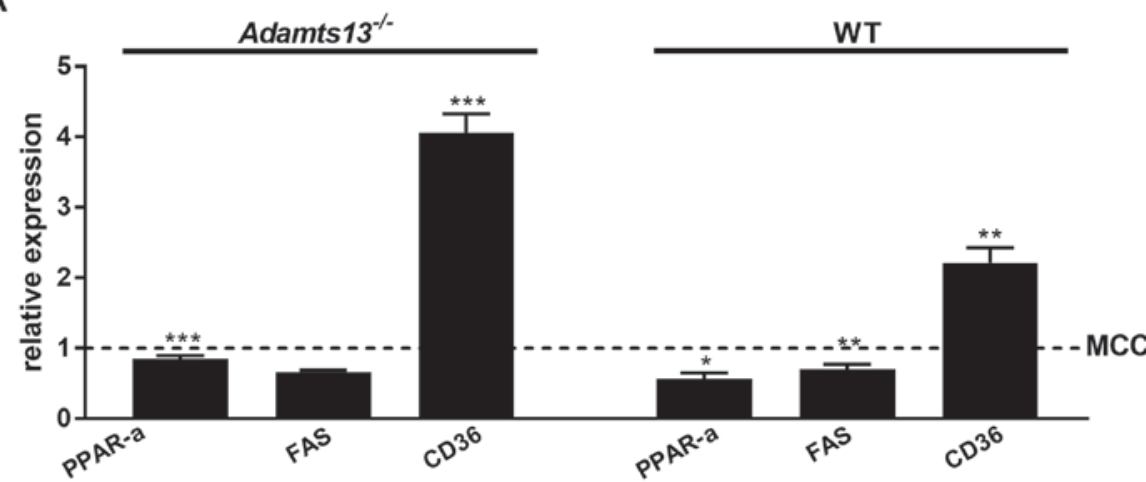

B

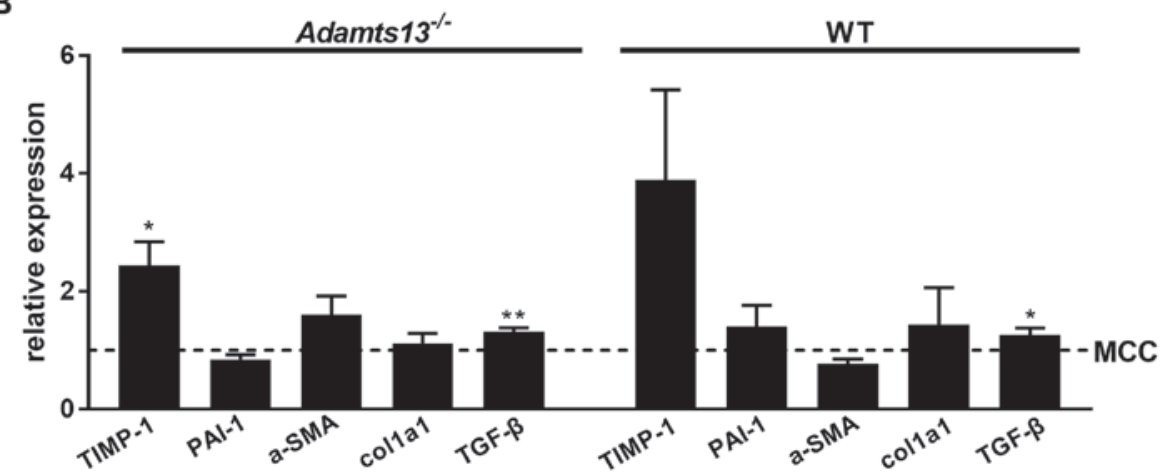

C

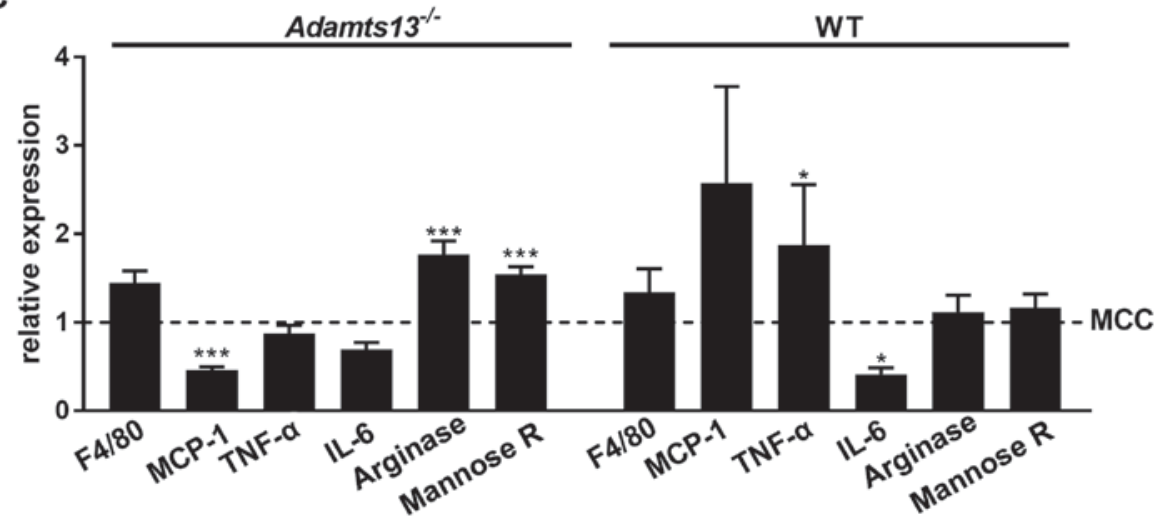

Figure 3. Effect of MCD diet on (A) expression of hepatic markers of steatosis/triglyceride metabolism, (B) fibrosis and (C) inflammation. Gene expression compared with the MCC diet is presented for MCD fed mice. Data are presented as the mean \pm standard error of $10\left(\right.$ Adamts $\left.13^{-/}\right)$or 5 (WT) experiments for each diet. ${ }^{*} \mathrm{P}<0.05,{ }^{* *} \mathrm{P}<0.01$ and ${ }^{* * * *} \mathrm{P}<0.001 \mathrm{MCD}$ vs. MCC. MCD, methionine and choline deficient diet; MCC, control diet; Adamts $13^{-1}$, a disintegrin and metalloproteinase with thrombospondin type-1 motif, member 13 deficient; WT, wild-type; PPAR- $\alpha$, peroxisome proliferator-activated receptor $\alpha$; FAS, Fas cell surface death receptor; CD36, cluster of differentiation; TIMP-1, tissue activator of metalloproteinase 1; PAI-1, plasminogen activator inhibitor 1; $\alpha$-SMA, $\alpha$-smooth muscle actin; Colla1, collagen type I $\alpha 1$ chain; TGF- $\beta$, transforming growth factor $\beta$; MCP-1, monocyte chemoattractant protein 1 ; TNF- $\alpha$, tumor necrosis factor $\alpha$; IL-6, interleukin 6.

score 1) mice exhibited only mild steatosis, whereas they all scored 0 for hepatocyte ballooning, lobular inflammation and fibrosis (Fig. 2A and Table III). However, following MCD feeding, WT and Adamts $13^{-/-}$mice presented with histological abnormalities with markedly enhanced scores for steatosis, ballooning, lobular inflammation and NAS without, however, significant differences between genotypes (Fig. 2C). In addition, fibrosis stage and score were deteriorated in MCD vs. MCC fed mice, however no clear effect of genotype was observed (Fig. 2B and Table III).

Relative expression of markers of triglyceride metabolism, fibrosis and inflammation were not markedly affected by genotype on either diet (data not shown). Histological observations on steatosis and fibrosis were compatible with markedly enhanced gene expression of the steatosis maker CD36 and the fibrosis marker TIMP-1 for MCD vs. MCC fed mice of the two genotypes (Fig. 3A and B). Analysis of hepatic inflammatory markers identified enhanced expression of TNF- $\alpha$ for WT mice on MCD as compared with MCC feeding (Fig. 3C).

\section{Discussion}

Deficiency of ADAMTS13, the VWF cleaving proteinase, results in thrombotic thrombocytopenic purpura (TTP), a 
rare however severe thrombotic disease (27). A functional role of ADAMTS13 has also been suggested in liver diseases, however the available data remain controversial (12). It has been previously demonstrated that ADAMTS13 antigen and activity levels are enhanced in obese mice, whereas ADAMTS13 deficiency does not affect development of NASH in obese mice $(28,29)$. In the present study, it was observed that a steatosis-inducing diet, independent of obesity, induces NASH to a comparable extent in Adamts 13 deficient and WT mice.

In patients, visceral obesity is frequently associated with NAFLD and NASH. These conditions may be associated with reduced ADAMTS13 activity, as reported in an obese patient with recurrent TTP; defective ADAMTS13 synthesis was suggested as a possible consequence of NASH (30). Furthermore, an apparent paradox with respect to the potential role of ADAMTS13 in development of acute liver failure has been recognized (12). ADAMTS13 activity has been identified to be inversely associated with disease severity and outcome, whereas by contrast, no ultralarge VWF multimers were identified in the systemic circulation, and high molecular weight VWF levels and VWF function were reduced in patients with acute liver failure as compared with healthy controls (31).

Due to the fact that NASH is difficult to study in humans due to slow progression of the disease and ethical considerations, animal models of $\mathrm{NASH}$ are crucial to improve the understanding of the pathogenesis of the disease. The model of feeding rodents with an MCD used in the present study is one of the most commonly used in NASH research (32-35). It is characterized by macrovesicular steatosis, hepatocellular death, inflammation, oxidative stress and fibrosis. Due to this diet, the mice lose weight and the metabolic profile is opposite to that of typical human NASH, e.g. the mice do not develop hyperlipidemia or hypertriglyceridemia and do not present with insulin resistance. However, liver injury and steatohepatitis are histologically similar to that of human patients (36-38). The severity of NASH induced in rodents by the MCD diet depends on the administration scheme, however is also affected by species, gender and the animal strain $(39,40)$. Using the administration scheme used in the present study (4 weeks of MCD/MCC), a protective effect of the ADAMTS5 deficiency (C57Bl/6J background) on development of NASH was identified (41). A similar administration scheme was used by Rinella et al (35). Although a time course was not performed, different effects of ADAMTS13 deficiency at earlier time points (e.g. two weeks) cannot be excluded.

It was reported that steatosis in mice kept on the MCD diet develops within 2-4 weeks and there is progression to fibrosis by $8-10$ weeks $(42-44)$. The mice in the current study developed steatosis on the 4-week diet, which was not restricted to centrolobular or periportal liver portions, however was diffusely spread throughout the liver tissue. More pronounced steatosis and higher liver triglyceride levels were identified upon MCD feeding of the two genotypes, however no differences between the genotypes were observed. No mice developed severe fibrosis, although mRNA expression of the fibrosis markers TIMP-1 and TGF- $\beta$ in the liver tissue were enhanced following the short-term MCD diet. These results were supported by similar hepatic expression profiles of steatosis and fibrosis markers for the two genotypes. The liver enzymes alkaline phosphatases, AST and ALT were enhanced on the MCD diet, indicating liver damage, however without marked differences between genotypes. Platelet counts appeared lower following MCD diet feeding, which was most pronounced in Adamts $13^{-/-}$mice. To the best of our knowledge, this has not been reported previously and remains to be fully elucidated.

In the present study, it was observed that the WT and the Adamts13 deficient mice fed with the MCD diet lose weight and present with low fasting blood glucose, however do not develop insulin resistance, which is in agreement with previous studies $(32,33,38)$. Improved insulin sensitivity upon MCD feeding of the two genotypes was observed, whereas HOMA-IR was lower for Adamts13 $3^{--}$vs. WT mice on either diet. By contrast, it has been previously observed that in mice with high fat induced obesity, glucose and insulin sensitivity did not differ between WT and Adamts13 $3^{-/-}$mice (28).

Taken together, the results of the present study and previous data from the literature (28) do not support a functional role for ADAMTS13 in the development of steatohepatitis in mouse models of diet-induced steatosis. This is in agreement with a recent study in patients with $\mathrm{NASH}$, demonstrating that plasma ADAMTS13 levels were not different from that of the controls (45).

\section{Acknowledgements}

The authors would like to thank Mrs. Liesbeth Frederix, Ms. Inge Vorsters and Ms. Christine Vranckx for technical support. The Center for Molecular and Vascular Biology and the Laboratory of Thrombosis Research are supported by the 'Programma financiering KU Leuven' (PF10/014). The current study was additionally supported by the 'Fonds voor Wetenschappelijk Onderzoek Vlaanderen' of the Flemish government (FWO grant G.0D13.15 N and KU Leuven grant OT/14/071 to Professor Karen Vanhoorelbeke).

\section{References}

1. Cassiman D and Jaeken J: NASH may be trash. Gut 57: 141-144, 2008.

2. Powell EE, Cooksley WG, Hanson R, Searle J, Halliday JW and Powell LW: The natural history of nonalcoholic steatohepatitis: A follow-up study of forty-two patients for up to 21 years. Hepatology 11: 74-80, 1990.

3. Harrison SA, Torgerson S and Hayashi PH: The natural history of nonalcoholic fatty liver disease: A clinical histopathological study. Am J Gastroenterol 98: 2042-2047, 2003.

4. Cohen JC, Horton JD and Hobbs HH: Human fatty liver disease: Old questions and new insights. Science 332: 1519-1523, 2011.

5. Bedogni G, Miglioli L, Masutti F, Tiribelli C, Marchesini G and Bellentani S: Prevalence of and risk factors for nonalcoholic fatty liver disease: The Dionysos nutrition and liver study. Hepatology 42: 44-52, 2005.

6. Blachier M, Leleu H, Peck-Radosavljevic M, Valla DC and Roudot-Thoraval F: The burden of liver disease in Europe: A review of available epidemiological data. J Hepatol 58: 593-608, 2013.

7. Sanyal AJ; American Gastroenterological Association: AGA technical review on nonalcoholic fatty liver disease. Gastroenterology 123: 1705-1725, 2002.

8. Neuschwander-Tetri BA: Nonalcoholic steatohepatitis and the metabolic syndrome. Am J Med Sci 330: 326-335, 2005.

9. Le Goff C and Cormier-Daire V: The ADAMTS(L) family and human genetic disorders. Hum Mol Genet 20: R163-R167, 2011. 
10. Uemura M, Tatsumi K, Matsumoto M,Fujimoto M, Matsuyama T, Ishikawa M, Iwamoto TA, Mori T, Wanaka A, Fukui $\mathrm{H}$ and Fujimura Y: Localization of ADAMTS13 to the stellate cells of human liver. Blood 106: 922-924, 2005.

11. Watanabe N, Ikeda H, Kume Y, Satoh Y, Kaneko M, Takai D, Tejima K, Nagamine M, Mashima H, Tomiya T, et al: Increased production of ADAMTS13 in hepatic stellate cells contributes to enhanced plasma ADAMTS13 activity in rat models of cholestasis and steatohepatitis. Thromb Haemost 102: 389-396, 2009.

12. Uemura M, Fujimura Y, Ko S, Matsumoto M, Nakajima $Y$ and Fukui H: Pivotal role of ADAMTS13 function in liver diseases. Int J Hematol 91: 20-29, 2010.

13. Takaya H, Uemura M, Fujimura Y, Matsumoto M, Matsuyama T, Kato S, Morioka C, Ishizashi $\mathrm{H}$, Hori Y, Fujimoto M, et al ADAMTS13 activity may predict the cumulative survival of patients with liver cirrhosis in comparison with the Child-Turcotte-Pugh score and the model for end-stage liver disease score. Hepatol Res 42: 459-472, 2012.

14. Uemura M, Matsuyama T, Ishikawa M, Fujimoto M, Kojima $\mathrm{H}$, Sakurai S, Ishii S, Toyohara M, Yamazaki M, Yoshiji H, et al: Decreased activity of plasma ADAMTS13 may contribute to the development of liver disturbance and multiorgan failure in patients with alcoholic hepatitis. Alcohol Clin Exp Res 29 (12 Suppl): 264S-271S, 2005

15. Matsumoto M, Kawa K, Uemura M, Kato S, Ishizashi $H$, Isonishi A, Yagi H, Park YD, Takeshima Y, Kosaka Y, et al: Prophylactic fresh frozen plasma may prevent development of hepatic VOD after stem cell transplantation via ADAMTS13-mediated restoration of von Willebrand factor plasma levels. Bone Marrow Transplant 40: 251-259, 2007.

16. Ikeda H, Tateishi R, Enooku K, Yoshida H, Nakagawa $H$ Masuzaki R, Kondo Y, Goto T, Shiina S, Kume Y, et al: Prediction of hepatocellular carcinoma development by plasma ADAMTS13 in chronic hepatitis B and C. Cancer Epidemiol Biomarkers Prev 20: 2204-2211, 2011.

17. Motto DG, Chauhan AK, Zhu G, Homeister J, Lamb CB, Desch KC, Zhang W, Tsai HM, Wagner DD and Ginsburg D: Shigatoxin triggers thrombotic thrombocytopenic purpura in genetically susceptible ADAMTS13-deficient mice. J Clin Invest 115: 2752-2761, 2005.

18. De Maeyer B, De Meyer SF, Feys HB, Pareyn I, Vandeputte N, Deckmyn $\mathrm{H}$ and Vanhoorelbeke K: The distal carboxyterminal domains of murine ADAMTS13 influence proteolysis of platelet-decorated VWF strings in vivo. J Thromb Haemost 8: 2305-2312, 2010

19. Council NR: Guide for the Care and Use Laboratoy Animals. 7th edition. Washington, DC, National Academy Press, 1996.

20. Kleiner DE, Brunt EM, Van Natta M, Behling C, Contos MJ, Cummings OW, Ferrell LD, Liu YC, Torbenson MS Unalp-Arida A, et al: Design and validation of a histological scoring system for nonalcoholic fatty liver disease. Hepatology 41: 1313-1321, 2005.

21. Verbeek J, Lannoo M, Pirinen E, Ryu D, Spincemaille P, Vander Elst I, Windmolders P, Thevissen K, Cammue BP, van Pelt J, et al: Roux-en-y gastric bypass attenuates hepatic mitochondrial dysfunction in mice with non-alcoholic steatohepatitis Gut 64: 673-683, 2015.

22. Bedossa P, Poitou C, Veyrie N, Bouillot JL, Basdevant A, Paradis V, Tordjman $\mathrm{J}$ and Clement K: Histopathologica algorithm and scoring system for evaluation of liver lesions in morbidly obese patients. Hepatology 56: 1751-1759, 2012.

23. Bedossa P, Dargère D and Paradis V: Sampling variability of liver fibrosis in chronic hepatitis C. Hepatology 38: 1449-1457, 2003.

24. Livak KJ and Schmittgen TD: Analysis of relative gene expression data using real-time quantitative PCR and the 2 (-Delta Delta C(T)) method. Methods 25: 402-408, 2001.

25. Verhenne S, Denorme F, Libbrecht S, Vandenbulcke A, Pareyn I, Deckmyn H, Lambrecht A, Nieswandt B, Kleinschnitz C, Vanhoorelbeke K and De Meyer SF: Platelet-derived VWF is not essential for normal thrombosis and hemostasis but fosters ischemic stroke injury in mice. Blood 126: 1715-1722, 2015.

26. Deforche L, Tersteeg C, Roose E, Vandenbulcke A, Vandeputte N, Pareyn I, De Cock E, Rottensteiner H, Deckmyn H, De Meyer SF and Vanhoorelbeke K: Generation of anti-murine ADAMTS13 antibodies and their application in a mouse model for acquired thrombotic thrombocytopenic purpura. PLoS One 11: e0160388, 2016.
27. Murrin RJ and Murray JA: Thrombotic thrombocytopenic purpura: Aetiology, pathophysiology and treatment. Blood Rev 20: 51-60, 2006

28. Geys L, Scroyen I, Roose E, Vanhoorelbeke K and Lijnen HR: ADAMTS13 deficiency in mice does not affect adipose tissue development. Biochim Biophys Acta 1850: 1368-1374, 2015.

29. Geys L, Bauters D, Roose E, Tersteeg C, Vanhoorelbeke K, Hoylaerts MF, Lijnen RH and Scroyen I: ADAMTS13 deficiency promotes microthrombosis in a murine model of diet-induced liver steatosis. Thromb Haemost 117: 19-26, 2017.

30. Lombardi AM, Fabris R, Berti de Marinis G, Marson P, Navaglia F, Plebani M, Vettor R and Fabris F: Defective ADAMTS13 synthesis as a possible consequence of NASH in an obese patient with recurrent thrombotic thrombocytopenic purpura. Eur J Haematol 92: 497-501, 2014.

31. Hugenholtz GC, Adelmeijer J, Meijers JC, Porte RJ, Stravitz RT and Lisman T: An unbalance between von Willebrand factor and ADAMTS13 in acute liver failure: Implications for hemostasis and clinical outcome. Hepatology 58: 752-761, 2013.

32. Takahashi Y, Soejima Y and Fukusato T: Animal models of nonalcoholic fatty liver disease/nonalcoholic steatohepatitis. World J Gastroenterol 18: 2300-2308, 2012.

33. Machado MV, Michelotti GA, Xie G, Almeida Pereira T, Boursier J, Bohnic B, Guy CD and Diehl AM: Mouse models of diet-induced nonalcoholic steatohepatitis reproduce the heterogeneity of the human disease. PLoS One 10: e0127991, 2015.

34. Caballero F, Fernández A, Matías N, Martínez L, Fucho R, Elena M, Caballeria J, Morales A, Fernández-Checa JC and García-Ruiz C: Specific contribution of methionine and choline in nutritional nonalcoholic steatohepatitis: Impact on mitochondrial S-adenosyl-L-methionine and glutathione. J Biol Chem 285: 18528-18536, 2010

35. Rinella ME, Elias MS, Smolak RR, Fu T, Borensztajn $\mathrm{J}$ and Green RM: Mechanisms of hepatic steatosis in mice fed a lipogenic methionine choline-deficient diet. J Lipid Res 49: 1068-1076, 2008

36. Weltman MD, Farrell GC and Liddle C: Increased hepatocyte CYP2E1 expression in a rat nutritional model of hepatic steatosis with inflammation. Gastroenterology 111: 1645-1653, 1996.

37. Weltman MD, Farrell GC, Hall P, Ingelman-Sundberg $M$ and Liddle C: Hepatic cytochrome P450 2E1 is increased in patients with nonalcoholic steatohepatitis. Hepatology 27: 128-133, 1998.

38. Rinella ME and Green RM: The methionine-choline deficient dietary model of steatohepatitis does not exhibit insulin resistance. J Hepatol 40: 47-51, 2004.

39. Fan JG and Qiao L: Commonly used animal models of non-alcoholic steatohepatitis. Hepatobiliary Pancreat Dis Int 8: 233-240, 2009.

40. Kirsch R, Clarkson V, Shephard EG, Marais DA, Jaffer MA, Woodburne VE, Kirsch RE and Hall Pde L: Rodent nutritional model of non-alcoholic steatohepatitis: Species, strain and sex difference studies. J Gastroenterol Hepatol 18: 1272-1282, 2003.

41. Bauters D, Spincemaille P, Geys L, Cassiman D, Vermeersch P, Bedossa P, Scroyen I and Lijnen RH: ADAMTS5 deficiency protects against non-alcoholic steatohepatitis (NASH) in obesity. Liver Int 36: 1848-1859, 2016

42. Dela Pena A, Leclercq I, Field J, George J, Jones B and Farrell G: NF-kappaB activation, rather than TNF, mediates hepatic inflammation in a murine dietary model of steatohepatitis. Gastroenterology 129: 1663-1674, 2005.

43. Leclercq IA, Farrell GC, Field J, Bell DR, Gonzalez FJ and Robertson GR: CYP2E1 and CYP4A as microsomal catalysts of lipid peroxides in murine nonalcoholic steatohepatitis. J Clin Invest 105: 1067-1075, 2000.

44. Ip E, Farrell G, Hall P, Robertson G and Leclercq I: Administration of the potent PPARalpha agonist, Wy-14,643, reverses nutritional fibrosis and steatohepatitis in mice. Hepatology 39: 1286-1296, 2004.

45. Potze W, Siddiqui MS, Boyett SL, Adelmeijer J, Daita K, Sanyal AJ and Lisman T: Preserved hemostatic status in patients with non-alcoholic fatty liver disease. J Hepatol 65: 980-987, 2016. 